\title{
Some algebraic aspects of Morse code sequences
}

\author{
Johann Cigler \\ Institut für Mathematik, Universität Wien, A-1090 Wien, Strudlhofgasse 4, Austria. \\ e-mail: Johann. Cigler@univie.ac.at
}

received Feb 25, 2003, accepted May 1, 2003.

\begin{abstract}
Morse code sequences are very useful to give combinatorial interpretations of various properties of Fibonacci numbers. In this note we study some algebraic and combinatorial aspects of Morse code sequences and obtain several $q$-analogues of Fibonacci numbers and Fibonacci polynomials and their generalizations.
\end{abstract}

Keywords: Fibonacci polynomial, $q$-analogue, $q$-binomial coefficient

\section{Morse code polynomials}

Morse code sequences are finite sequences of dots $(\bullet)$ and dashes $(-)$. If a dot has length 1 and a dash has length 2 then the number of all such sequences of total length $n-1$ is the Fibonacci number $F_{n}$, which is defined as the sequence of numbers satisfying the recursion $F_{n}=F_{n-1}+F_{n-2}$ with initial conditions $F_{0}=0$ and $F_{1}=1$. If a dash is assumed to have length $j, j \geq 1$, we get a simple generalization of Fibonacci numbers.

Let $M C$ be the set of all Morse code sequences. We interpret $M C$ as a monoid with respect to concatenation whose unit element is the empty sequence $\varepsilon$. If we write $a$ for a dot and $b$ for a dash then $M C$ consists of all words in $a$ and $b$. Let $P$ be the corresponding monoid algebra over $\mathbb{C}$, i. e. the algebra of all finite sums $\sum_{v \in M C} \lambda_{v} v$ with complex coefficients. The elements of $P$ will be called Morse code polynomials.

An important element of $P$ is the binomial

$$
(a+b)^{n}=\sum_{k=0}^{n} C_{k}^{n}(a, b)
$$

Here $C_{k}^{n}(a, b)$ is the sum of all words with $k$ dashes and $n-k$ dots. It is characterized by the boundary values $C_{k}^{0}(a, b)=\delta_{k, 0}$ and $C_{0}^{n}(a, b)=a^{n}$ and each of the two recursions

$$
C_{k}^{n+1}(a, b)=b C_{k-1}^{n}(a, b)+a C_{k}^{n}(a, b)
$$

or

$$
C_{k}^{n+1}(a, b)=C_{k-1}^{n}(a, b) b+C_{k}^{n}(a, b) a .
$$

1365-8050 (c) 2003 Discrete Mathematics and Theoretical Computer Science (DMTCS), Nancy, France 
It is clear that the image of $C_{k}^{n}(a, b)$ under the homomorphism $\varphi: P \rightarrow \mathbb{C}$, defined by $\varphi(a)=\varphi(b)=1$, is the binomial coefficient $\left(\begin{array}{l}n \\ k\end{array}\right)$.

By considering various other homomorphisms of this algebra into other algebras we obtain generalizations and $q$-analogues of the binomial coefficients.

We state some simple examples:

(a) Consider the homomorphism $\phi: P \rightarrow \mathbb{C}[x, s]$ defined by $\phi(a)=x, \phi(b)=s$, where $x$ and $s$ are commuting variables. Then we get of course $\phi\left(C_{k}^{n}(a, b)\right)=\left(\begin{array}{l}n \\ k\end{array}\right) x^{n-k} s^{k}$.

(b) Let $R$ be the ring of linear operators on the vector space of polynomials $\mathbb{C}[x, s]$. We will only use multiplication operators with

polynomials and the shift operators $\eta=\eta_{q}$ in $R$ defined by

$$
\eta f(x, s)=f(x, q s)
$$

for a positive real number $q$.

For each integer $j \geq 1$ we define homomorphisms $\varphi_{j}: P \rightarrow R$ by

$$
\varphi_{j}(a)=r(s) \eta, \quad \varphi_{j}(b)=t(s) \eta^{j},
$$

where $r(s)$ and $t(s)$ are polynomials in $\mathbb{C}[x, s]$. Then

$$
\varphi_{j}\left(C_{k}^{n}(a, b)\right)=a_{n, k, j}(s) \eta^{n+(j-1) k}
$$

for some polynomial $a_{n, k, j}(s)$.

From (1.2) we get

$$
a_{n, k, j}(s)=r(s) a_{n-1, k, j}(q s)+t(s) a_{n-1, k-1, j}\left(q^{j} s\right)
$$

and from (1.3)

$$
a_{n, k, j}(s)=r\left(q^{n-1+k(j-1)} s\right) a_{n-1, k, j}(s)+t\left(q^{n-1+(k-1)(j-1)} s\right) a_{n-1, k-1, j}(s) .
$$

For some special choices of $r(s)$ and $t(s)$ we can obtain explicit formulas for $a_{n, k, j}(s)$.

(b1) Frequent use will be made of the homomorphisms $\Phi_{j}$ with $r(s)=x$ and arbitrary $t(s)$. There are no explicit formulas known in this case.

(b2) For $r(s)=x$ and $t(s)=s$ we call the corresponding homomorphism $\Psi_{j}$ the Carlitz homomorphism, because it will play a fundamental role in the study of Carlitz's $q$-Fibonacci polynomials. It satisfies

$$
x \eta \cdot s \eta^{j}=q s \eta^{j} \cdot x \eta
$$

or in other words

$$
\Psi_{j}(a) \Psi_{j}(b)=q \Psi_{j}(b) \Psi_{j}(a) .
$$


We need the Gaussian $q$-binomial coefficients $\left[\begin{array}{l}n \\ k\end{array}\right]$ (cf. e. g. [2], [5] or [6]). We define them by

$$
\left[\begin{array}{l}
n \\
k
\end{array}\right]=\prod_{i=1}^{k} \frac{q^{n-i+1}-1}{q^{i}-1} \quad \text { for } n, k \in \mathbb{N} \text {. }
$$

They satisfy the following recursions

$$
\left[\begin{array}{c}
n+1 \\
k
\end{array}\right]=q^{k}\left[\begin{array}{l}
n \\
k
\end{array}\right]+\left[\begin{array}{c}
n \\
k-1
\end{array}\right]
$$

and

$$
\left[\begin{array}{c}
n+1 \\
k
\end{array}\right]=\left[\begin{array}{l}
n \\
k
\end{array}\right]+q^{n-k+1}\left[\begin{array}{c}
n \\
k-1
\end{array}\right]
$$

The $q$-binomial theorem (see e. g. [2], [5] or [6]) states that for $n \in \mathbb{N}$

$$
(A+B)^{n}=\sum_{k \geq 0}\left[\begin{array}{l}
n \\
k
\end{array}\right] B^{k} A^{n-k} \quad \text { if } \quad A B=q B A .
$$

Therefore we get

$$
\Psi_{j}\left(C_{k}^{n}(a, b)\right)=\left[\begin{array}{l}
n \\
k
\end{array}\right]\left(s \eta^{j}\right)^{k}(x \eta)^{n-k} .
$$

The corresponding polynomials are

$$
a_{n, k, j}(s)=q^{j\left(\begin{array}{l}
k \\
2
\end{array}\right)}\left[\begin{array}{l}
n \\
k
\end{array}\right] x^{n-k} s^{k}
$$

(b3) If we choose $r(s)=x \cdot(1+s), t(s)=s$ and $j=2$ we have

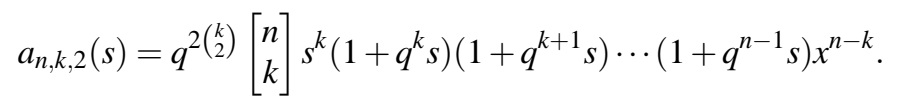

We prove this by induction. For $n=0$ and all $k$ it is trivially true. Suppose it holds for $n$ and all $k$. By (1.6) we have to show

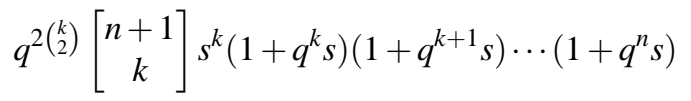

$$
\begin{aligned}
& =(1+s) q^{2\left(\begin{array}{l}
k \\
2
\end{array}\right)}\left[\begin{array}{l}
n \\
k
\end{array}\right](q s)^{k}\left(1+q^{k+1} s\right) \cdots\left(1+q^{n} s\right) \\
& +s q^{2\left(\begin{array}{c}
k-1 \\
2
\end{array}\right)}\left[\begin{array}{c}
n \\
k-1
\end{array}\right]\left(q^{2} s\right)^{k}\left(1+q^{k+1} s\right) \cdots\left(1+q^{n+1} s\right)
\end{aligned}
$$


or equivalently

$$
\left[\begin{array}{c}
n+1 \\
k
\end{array}\right]\left(1+q^{k} s\right)=(1+s) q^{k}\left[\begin{array}{l}
n \\
k
\end{array}\right]+\left[\begin{array}{c}
n \\
k-1
\end{array}\right]\left(1+q^{n+1} s\right) .
$$

Comparing coefficients this is equivalent with the recursions of the $q$-binomial coefficients.

This homomorphism will be used in our treatment of the Al-Salam and Ismail polynomials (cf. [⿴囗十], [10]).

\section{Abstract Fibonacci polynomials}

Now we want to define abstract Fibonacci polynomials of order $j \geq 1$. To this end we consider again the algebra generated by the Morse code sequences. For each word $w$ in $M C$ we define a length $l(j, w)$ by $l(j, a)=1, l(j, b)=j$ and $l\left(j, w_{1} w_{2}\right)=l\left(j, w_{1}\right)+l\left(j, w_{2}\right)$. We define these polynomials $F_{n}(j, a, b)$ as the sum of all monomials $w \in M C$ of length $l(j, w)=n-j+1$.

Then it is clear that

$$
F_{n}(j, a, b)=a F_{n-1}(j, a, b)+b F_{n-j}(j, a, b)
$$

and also

$$
F_{n}(j, a, b)=F_{n-1}(j, a, b) a+F_{n-j}(j, a, b) b
$$

with initial values

$$
F_{0}(j, a, b)=\cdots=F_{j-2}(j, a, b)=0, F_{j-1}(j, a, b)=\varepsilon .
$$

For $j=1$ we have of course $F_{n}(1, a, b)=(a+b)^{n}$ and for $j=2$ we get the abstract Fibonacci polynomials introduced in [7].

A word $w$ of length $l(j, w)=n-j+1$ with $k$ dashes consists of $n-(j-1)(k+1)$ letters and all these occur in $F_{n}(j, a, b)$. Therefore we have

Theorem 2.1. The abstract Fibonacci polynomials of order $j \geq 1$ are given by

$$
F_{n}(j, a, b)=\sum_{0 \leq k \leq \frac{n-j+1}{j}} C_{k}^{n-(j-1)(k+1)}(a, b) .
$$

By applying the homomorphism $\varphi_{j}$ we get

$$
\varphi_{j}\left(F_{n}(j, a, b)\right)=r(s) \eta \varphi_{j}\left(F_{n-1}(j, a, b)\right)+t(s) \eta^{j} \varphi_{j}\left(F_{n-j}(j, a, b)\right)
$$

or equivalently

$$
\varphi_{j}\left(F_{n}(j, a, b)\right)=\varphi_{j}\left(F_{n-1}(j, a, b)\right) r(s) \eta+\varphi_{j}\left(F_{n-j}(j, a, b)\right) t(s) \eta^{j} .
$$

This implies that

$$
\varphi_{j}\left(F_{n}(j, a, b)\right)=F_{n}^{*}(j, s, q) \eta^{n-j+1},
$$

where the polynomials $F_{n}^{*}(j, s, q)$, which we call general $q$-Fibonacci polynomials satisfy the recurrence

$$
F_{n}^{*}(j, s, q)=r(s) F_{n-1}^{*}(j, q s, q)+t(s) F_{n-j}^{*}\left(j, q^{j} s, q\right)
$$


or equivalently

$$
F_{n}^{*}(j, s, q)=r\left(q^{n-j} s\right) F_{n-1}^{*}(j, s, q)+t\left(q^{n-2 j+1} s\right) F_{n-j}^{*}(j, s, q) .
$$

The most important special case is given by $r(s)=x$ and $t(s)=s$. The corresponding polynomials $F_{n}(j, x, s, q)$ will be called Carlitz-Fibonacci polynomials of order $j$, because Carlitz has studied the special case $j=2$.

Theorem 2.2. The Carlitz-Fibonacci polynomials of order $j$ satisfy the recursion

$$
F_{n}(j, x, s, q)=x F_{n-1}(j, x, s, q)+q^{n-2 j+1} s F_{n-j}(j, x, s, q)
$$

or equivalently

$$
F_{n}(j, x, s, q)=x F_{n-1}(j, x, q s, q)+s F_{n-j}\left(j, x, q^{j} s, q\right)
$$

with initial values

$$
F_{0}(j, x, s, q)=\cdots=F_{j-2}(j, x, s, q)=0, \quad F_{j-1}(j, x, s, q)=1 .
$$

They are given by

$$
F_{n}(j, x, s, q)=\sum_{0 \leq k j \leq n-j+1} q^{j\left(\begin{array}{l}
k \\
2
\end{array}\right)}\left[\begin{array}{c}
n-(j-1)(k+1) \\
k
\end{array}\right] s^{k} x^{n-(k+1) j+1} .
$$

For $j=2$ these polynomials have been studied in [4] and [7]. The formula (2.10) follows immediately from (2.4) and (1.9).

\section{Some examples.}

(a) For $j=1$ we get by applying the $q$-binomial theorem

(1.8)

the well-known formula

$$
F_{n}(1, x, s, q)=(x+s)(x+q s) \cdots\left(x+q^{n-1} s\right)=\sum\left[\begin{array}{l}
n \\
k
\end{array}\right] q^{\left(\begin{array}{c}
k \\
2
\end{array}\right) s^{k} x^{n-k}}
$$

(b) Let $E_{n}=E_{n-1}-q^{n} E_{n-j}$ with initial values $E_{0}=\cdots=E_{j-1}=1$ and $D_{n}=D_{n-1}-q^{n} D_{n-j}$ with initial values $D_{i}=1-q[i], i=0, \ldots, j-1$, where $[i]$ denotes $[i]=\frac{q^{i}-1}{q-1}$. It is clear that $E_{n}=$ $F_{n+j-1}\left(j, 1,-q^{j}, q\right)$.

Therefore from (2.10) we get

$$
E_{n}=\sum_{0 \leq k j \leq n}\left[\begin{array}{c}
n-(j-1) k \\
k
\end{array}\right] q^{j\left(\begin{array}{c}
(k+1 \\
2
\end{array}\right)(-1)^{k}}
$$

which has been shown in [I]].

Furthermore $D_{n}=F_{n+2 j-2}(j, 1,-q, q)$, which gives the second formula shown in [W]

$$
D_{n}=\sum_{0 \leq k j \leq n+j-1}\left[\begin{array}{c}
n-(j-1)(k-1) \\
k
\end{array}\right](-1)^{k} q^{k+j\left(\begin{array}{l}
k \\
2
\end{array}\right) .}
$$


(c) If we set $t(s)=1$ and $r\left(q^{k} s\right)=x_{k+1}$ we get the continuants studied in [9].

(d) If we choose $j=2$ and $r(s)=\xi(1+s), t(s)=-\beta s$ for some constants $\beta$, $\xi$ and denote the corresponding homomorphism $\chi$ then

$$
F_{n+1}^{*}(2, s)=\chi\left(F_{n+1}(2, a, b)\right) 1=U_{n}\left(\xi, s, \frac{\beta}{s}\right),
$$

where the $U_{n}(\xi, s, \beta)$ are the Al-Salam and Ismail polynomials (cf. [U] or [10]), since the recurrence and the initial values coincide.

From (2.4) we get immediately that

$$
U_{n}(\xi, \sigma, \beta)=\sum_{0 \leq 2 k \leq n} q^{2\left(\begin{array}{c}
k \\
2
\end{array}\right)}\left[\begin{array}{c}
n-k \\
k
\end{array}\right](-\beta)^{k}\left(1+q^{k} \sigma\right) \cdots\left(1+q^{n-k} \sigma\right) \xi^{n-2 k},
$$

which is formula (1.12) of [10].

(e) A further interesting example occurs by choosing the homomorphism $\psi$ of $P$ into the algebra of operators on $\mathbb{C}[x, s]$ defined by

$$
\psi(a)=x+(q-1) s D, \quad \psi(b)=s, \quad \text { where } \quad D h(x)=\frac{h(q x)-h(x)}{q x-x}
$$

is the operator of $q$-differentiation. The corresponding Fibonacci polynomials $F i b_{n}(x, s)$ have been studied in [8]. They satisfy the recurrence

$$
F i b_{n}(x, s)=x F i b_{n-1}(x, s)+(q-1) s{ }^{2} F i b_{n-1}(x, s)+s F i b_{n-2}(x, s)
$$

with initial values $F i b_{0}(x, s)=0, F i b_{1}(x, s)=1$.

They are explicitly given by

$$
F_{i b}(x, s)=\sum_{k=0}^{\left\lfloor\frac{n}{2}\right\rfloor}\left[\begin{array}{c}
n-k-1 \\
k
\end{array}\right] q^{\left(\frac{k+1}{2}\right)} x^{n-1-2 k} s^{k} .
$$

For the proof it suffices to compare coefficients. This leads to the identity

$$
q^{\left(\begin{array}{c}
k+1 \\
2
\end{array}\right)}\left[\begin{array}{c}
n-k \\
k
\end{array}\right]=q^{\left(\begin{array}{c}
k+1 \\
2
\end{array}\right)}\left[\begin{array}{c}
n-k-1 \\
k
\end{array}\right]+(q-1) q^{\left(\begin{array}{l}
k \\
2
\end{array}\right)}\left[\begin{array}{l}
n-k \\
k-1
\end{array}\right][n-2 k+1]+q^{\left(\begin{array}{c}
k \\
2
\end{array}\right)}\left[\begin{array}{c}
n-k-1 \\
k-1
\end{array}\right]
$$

which is easily verified. Here $[n]$ denotes $[n]=\frac{q^{n}-1}{q-1}$.

Remark. Whereas for the Carlitz-Fibonacci polynomials (2.10) no $q$-analogue of the Lucas polynomials with a simple recurrence exists, we have in this case a precise $q$-analogue (cf. [8]). It satisfies the same recurrence

$$
\operatorname{Luc}_{n}(x, s)=(x+(q-1) s D) \operatorname{Luc}_{n-1}(x, s)+s \operatorname{Luc} c_{n-2}(x, s)
$$

but with initial values $\operatorname{Luc}_{0}(x, s)=2, \operatorname{Luc}_{1}(x, s)=x$ and is given by the explicit formula

$$
\operatorname{Luc}_{n}(x, s)=\sum_{j=0}^{\left\lfloor\frac{n}{2}\right\rfloor} \frac{[n]}{[n-j]}\left[\begin{array}{c}
n-j \\
j
\end{array}\right] q^{\left(\begin{array}{l}
j \\
2
\end{array}\right) x^{n-2 j} s^{j}}
$$




\section{A useful matrix}

Let

$$
C:=\left(\begin{array}{ll}
0 & \varepsilon \\
b & a
\end{array}\right)
$$

Then it is easily shown by induction that

$$
C^{n}=\left(\begin{array}{cc}
F_{n-1}(2, a, b) b & F_{n}(2, a, b) \\
F_{n}(2, a, b) b & F_{n+1}(2, a, b)
\end{array}\right) .
$$

As a special case we see that

$$
C^{2}=\left(\begin{array}{cc}
b & a \\
a b & a^{2}+b
\end{array}\right)=a C+b I
$$

where $I=\left(\begin{array}{cc}\varepsilon & 0 \\ 0 & \varepsilon\end{array}\right)$ is the identity matrix in the ring of matrices over $P$.

If we assume $b$ to be invertible then (3.3) can be used to show that $C$ is invertible and given by

$$
C^{-1}=b^{-1} C-b^{-1} a I=\left(\begin{array}{cc}
-b^{-1} a & b^{-1} \\
\varepsilon & 0
\end{array}\right) .
$$

One checks then that (3.2) holds for all $n \in \mathbb{Z}$. We may therefore also extend $F_{n}(2, a, b)$ to negative $n$ and obtain

$$
F_{-n}(2, a, b)=(-1)^{n-1} b^{-1} F_{n}\left(2, a b^{-1}, b^{-1}\right)=(-1)^{n-1} F_{n}\left(2, b^{-1} a, b^{-1}\right) b^{-1} .
$$

From $C^{-n} C^{n}=I$ and $C^{n} C^{-n}=I$ we may deduce a general form of the Cassini identities

$$
b F_{n+1}\left(2, b^{-1} a, b^{-1}\right) F_{n-1}(2, a, b)-F_{n}\left(2, a b^{-1}, b^{-1}\right) F_{n}(2, a, b)=(-1)^{n} \varepsilon
$$

and

$$
F_{n-1}(2, a, b) F_{n+1}\left(2, a b^{-1}, b^{-1}\right) b-F_{n}(2, a, b) F_{n}\left(2, b^{-1} a, b^{-1}\right)=(-1)^{n} \varepsilon .
$$

For the Fibonacci numbers we have the formula $F_{2 n}=\sum\left(\begin{array}{l}n \\ k\end{array}\right) F_{n-k}$. We now give an interesting generalization.

Theorem 3.1. For positive $n$ we get the following formula

$$
C^{2 n}=\sum_{k=0}^{n} C_{k}^{n}(a, b) C^{n-k}
$$


Proof. This holds for $n=1$. If it is already known for $n$ the following reasoning gives the result for $n+1$ :

$$
\begin{aligned}
C^{2 n+2} & =\sum_{k=0}^{n} C_{k}^{n}(a, b) C^{2} C^{n-k}=\sum_{k=0}^{n} C_{k}^{n}(a, b)(a C+b I) C^{n-k} \\
& =\sum_{k=0}^{n} C_{k}^{n}(a, b) a C^{n-k+1}+\sum_{k=0}^{n} C_{k}^{n}(a, b) b C^{n-k} \\
& =\sum_{k=0}^{n}\left(C_{k}^{n}(a, b) a+C_{k-1}^{n}(a, b) b\right) C^{n+1-k}=\sum_{k=0}^{n+1} C_{k}^{n+1}(a, b) C^{n+1-k}
\end{aligned}
$$

This is a somewhat curious formula. It means in fact that we may compute $(a C+b I)^{n}$ by first treating $C$ as an indeterminate commuting with both $a$ and $b$, and then writing the $C^{\prime} s$ at the rightmost place and interpret the result as a matrix over $P$.

By multiplying both sides from the right with $C^{m}$ and comparing coefficients we deduce from (3.5) that

$$
F_{2 n+m}(2, a, b)=\sum_{k=0}^{n} C_{k}^{n}(a, b) F_{m+n-k}(2, a, b) .
$$

In the same way we get for the transposed matrix

$$
D:=\left(\begin{array}{ll}
0 & b \\
\varepsilon & a
\end{array}\right)
$$

the formulas

$$
D^{n}=\left(\begin{array}{cc}
b F_{n-1}(2, a, b) & b F_{n}(2 a, b) \\
F_{n}(2, a, b) & F_{n+1}(2, a, b)
\end{array}\right)
$$

and

$$
D^{2 n}=\sum_{k=0}^{n} D^{n-k} C_{k}^{n}(a, b)
$$

From this we deduce as above

$$
F_{m+2 n}(2, a, b)=\sum F_{m+n-k}(2, a, b) C_{k}^{n}(a, b) .
$$

Remark. These formulas have an obvious combinatorial interpretation:

$F_{m+2 n}(2, a, b)$ is the sum of all words in $M C$ of length $m+2 n-1$. For $m>0$ each such word $w$ contains at least $n$ letters from the alphabet $\{a, b\}$ and can therefore be split into a product $w=u v$, where $u$ has precisely $n$ letters. If $u$ contains $k$ dashes then it has length $n+k$.

Therefore $v$ has length $m+n-k-1$.

On the other hand for each $k, 0 \leq k \leq n$, each $u_{k}$ with $k$ dashes and $n-k$ dots, and each word $v_{k}$ of length $m+n-k-1$ the word $u_{k} v_{k}$ has length $m+2 n-1$. Thus we have

$$
F_{m+2 n}(2, a, b)=\sum_{k} \sum_{u_{k}} u_{k} \sum_{v_{k}} v_{k}=\sum C_{k}^{n}(a, b) F_{m+n-k}(2, a, b) .
$$


For $m=0$ and $k=n$ the result is also true because $F_{0}(2, a, b)=0$. If we split the word $w$ in the form $w=v u$ we get the second formula.

If we apply $\Psi_{2}$ to (3.10) and remember (2.5) and (1.9) we get

$$
F_{m+2 n}(2, x, s, q)=\sum\left[\begin{array}{l}
n \\
k
\end{array}\right] q^{k(m+n-2)} s^{k} x^{n-k} F_{m+n-k}(2, x, s, q) .
$$

A special case of this formula has also been considered by Andrews [3]].

In an analogous way we deduce from (3.6) and (1.5) the formula

$$
F_{m+2 n}(2, x, s, q)=\sum\left[\begin{array}{l}
n \\
k
\end{array}\right] q^{2\left(\begin{array}{l}
k \\
2
\end{array}\right) s^{k} x^{n-k} F_{m+n-k}\left(2, x, q^{n+k} s, q\right)}
$$

In the same manner we get after some calculation the following doubling formula for the Al-Salam and Ismail polynomials:

$$
U_{m+2 n}(x ; a, b)=\sum U_{m+n-k}(x ; a, b) q^{k(m+n-1)}\left[\begin{array}{l}
n \\
k
\end{array}\right](-b)^{k}\left(1+q^{m+n} a\right) \cdots\left(1+q^{m+2 n-k-1} a\right) x^{n-k} .
$$

\section{The general case}

The generalizations for $j>2$ are straightforward. We have to consider now the $j \times j$-matrix

$$
C=\left(\begin{array}{ccccc}
0 & \varepsilon & 0 & \ldots & 0 \\
0 & 0 & \varepsilon & \ldots & 0 \\
\ldots & \ldots & \ldots & \ldots & \ldots \\
0 & 0 & 0 & \ldots & \varepsilon \\
b & 0 & 0 & \ldots & a
\end{array}\right)
$$

It is easily seen that

$$
C^{j}=a C^{j-1}+b I
$$

and that

$$
C^{j n}=\sum C_{k}^{n}(a, b) C^{(j-1)(n-k)} .
$$

For the transposed matrix $D=C^{t}$ we obtain analogously

$$
D^{j}=D^{j-1} a+I b
$$

and

$$
D^{j n}=\sum D^{(j-1)(n-k)} C_{k}^{n}(a, b) .
$$

From these formulas we deduce

$$
F_{m+j n}(j, x, s, q)=\sum\left[\begin{array}{l}
n \\
k
\end{array}\right] q^{j\left(\begin{array}{l}
k \\
2
\end{array}\right)+k(j-1)(n-k)+k(m-j+1)} s^{k} x^{n-k} F_{m+(j-1)(n-k)}(j, x, s, q)
$$

and

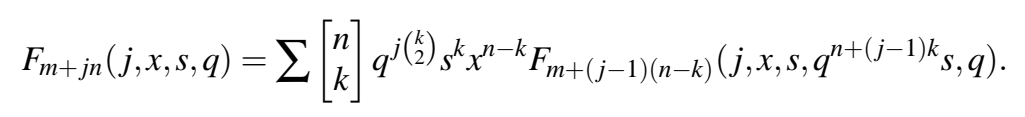


Remark. It is also easy to prove (4.5) by comparing coefficients of the powers of $x$. It is then equivalent to the $q$-Vandermonde identity

$$
\left[\begin{array}{c}
p+j n \\
k
\end{array}\right]=\sum\left[\begin{array}{l}
n \\
i
\end{array}\right]\left[\begin{array}{c}
p+(j-1) n \\
k-1
\end{array}\right] q^{i(p+(j-1) n-(k-i))}
$$

(cf. e. g. [5]).

If we start from the companion formula obtained by switching the factors

$$
\left[\begin{array}{c}
p+j n \\
k
\end{array}\right]=\sum\left[\begin{array}{l}
n \\
i
\end{array}\right]\left[\begin{array}{c}
p+(j-1) n \\
k-1
\end{array}\right] q^{(k-i)(n-i)}
$$

we get (4.6).

Both (4.5) and (4.6) can also be verified by induction with respect to $n$.

It is instructive to verify (4.5) or (4.6) for $j=1$.

L. Carlitz [4] has shown that

$$
x^{n} F_{m}(2, x, s, q)=\sum(-1)^{k}\left[\begin{array}{l}
k \\
n
\end{array}\right] q^{k(m-1)-\left(\begin{array}{c}
k+1 \\
2
\end{array}\right) s^{k} F_{m+n-2 k}(2, x, s, q)}
$$

holds for all $m$ and $n$. I shall give another proof of this fact and generalize it to arbitrary $j$ :

Theorem 4.1. The Carlitz-Fibonacci polynomials satisfy

$$
x^{n} F_{m}(j, x, s, q)=\sum_{k \in \mathbb{Z}}(-1)^{k}\left[\begin{array}{l}
n \\
k
\end{array}\right] q^{k(m-j+1)-(j-1)\left(\begin{array}{c}
k+1 \\
2
\end{array}\right) s^{k} F_{m+n-j k}(j, x, s, q) .}
$$

Proof. Let $\mathbb{C}\left[z, z^{-1}\right]$ be the vector space of all polynomials in $z$ and $\frac{1}{z}$. There we consider the linear operators $T, R_{j}, e$ defined by

$$
T z^{n}=z^{n+1}, \quad R_{j} z^{n}=z^{n+1-j}, \quad e z^{n}=q^{n} z^{n} .
$$

They satisfy the $q$-commutation rule $R_{j} e \cdot T=q T \cdot R_{j} e$ because for each $n \in \mathbb{Z}$ we have

$$
R_{j} e \cdot T z^{n}=R_{j} e z^{n+1}=q^{n+1} z^{n+2-j}
$$

and

$$
q T \cdot R_{j} e z^{n}=q^{n+1} T z^{n+1-j}=q^{n+1} z^{n+2-j} .
$$

From the $q$-binomial theorem we get therefore

$$
\left(T-q^{1-j} s R_{j} e\right)^{n}=\sum(-1)^{k}\left[\begin{array}{l}
n \\
k
\end{array}\right] T^{n-k}\left(q^{1-j} s R_{j} e\right)^{k} .
$$

This implies

$$
\begin{aligned}
\left(T-q^{1-j} s R_{j} e\right)^{n} z^{m} & =\sum(-1)^{k}\left[\begin{array}{l}
n \\
k
\end{array}\right] T^{n-k}\left(q^{1-j} s R_{j} e\right)^{k} z^{m} \\
& =\sum(-1)^{k}\left[\begin{array}{l}
n \\
k
\end{array}\right] q^{k(m-j+1)-(j-1)\left(\begin{array}{c}
k+1 \\
2
\end{array}\right) s^{k} z^{m+n-j k}}
\end{aligned}
$$


Now consider the linear map $\sigma$, defined by $\sigma\left(z^{n-j+1}\right)=f_{n}$ for all $n \in \mathbb{Z}$, where $f_{n}=\alpha_{n}$ with an indeterminate $\alpha_{n}$ if $F_{n}(j, s, x, q)=0$ and $f_{n}=F_{n}(j, x, s, q)$ else, in order to get a linearly independent set of vectors. Let $H$ be the vector space generated by the $f_{n}$, such that $\sigma$ becomes a bijection onto $H$. Then

$$
\sigma\left(T-q^{1-j} s R_{j} e\right) \sigma^{-1} f_{n}=\sigma\left(T-q^{1-j} s R_{j} e\right) z^{n-j+1}=f_{n+1}-s q^{n-2 j+2} f_{n+1-j}
$$

for all $n \in \mathbb{Z}$. The homomorphism $f_{n} \rightarrow F_{n}(j, x, s, q)$ maps the right hand side unto $x F_{n}(j, x, s, q)$. Therefore the kernel contains all $f_{n}$ with $F_{n}(j, x, s, q)=0$ and our theorem is proved.

Remark. For $m=0$ we get as special case of (4.7) that

$$
0=\sum(-1)^{k}\left[\begin{array}{l}
n \\
k
\end{array}\right] q^{-k-\left(\begin{array}{c}
k+1 \\
2
\end{array}\right) s^{k} F_{n-2 k}(2, x, s, q) .}
$$

Whereas for $q=1$ the terms $(-s)^{k} F_{n-2 k}(x, s, 1)+(-s)^{n-k} F_{2 k-n}(x, s, 1)$ vanish, this is not the case for the corresponding terms for $q \neq 1$.

\section{General $q$-Fibonacci polynomials}

Finally we want to generalize some results of [7] to the general $q$-Fibonacci polynomials $F_{n}^{*}(j, s, q)$, which satisfy the recurrence

$$
F_{n}^{*}(j, s, q)=r(s) F_{n-1}^{*}(j, q s, q)+t(s) F_{n-j}^{*}\left(j, q^{j} s, q\right)
$$

or equivalently

$$
F_{n}^{*}(j, s, q)=r\left(q^{n-j} s\right) F_{n-1}^{*}(j, s, q)+t\left(q^{n-2 j+1} s\right) F_{n-j}^{*}(j, s, q)
$$

with initial conditions

$$
F_{0}^{*}(j, s, q)=\cdots=F_{j-2}^{*}(j, s, q)=0, \quad F_{j-1}^{*}(j, s, q)=1 .
$$

Consider first the case $j=2$. Let

$$
A(s)=\left(\begin{array}{cc}
0 & 1 \\
t\left(\frac{s}{q}\right) & r(s)
\end{array}\right)
$$

and

$$
M_{n}(s)=A\left(q^{n-1} s\right) A\left(q^{n-2} s\right) \cdots A(s) .
$$

Then we get

$$
M_{k+n}(s)=M_{k}\left(q^{n} s\right) M_{n}(s)
$$

and

$$
M_{n}(s)=\left(\begin{array}{cc}
t\left(\frac{s}{q}\right) F_{n-1}^{*}(q s, q) & F_{n}^{*}(s, q) \\
t\left(\frac{s}{q}\right) F_{n}^{*}(q s, q) & F_{n+1}^{*}(s, q)
\end{array}\right) .
$$

Taking determinants we get the Cassini identity

$$
F_{n-1}^{*}(q s, q) F_{n+1}^{*}(s, q)-F_{n}^{*}(q s, q) F_{n}^{*}(s, q)=(-1)^{n} t(s) t(q s) \cdots t\left(q^{n-2} s\right) .
$$


If we extend (5.3) to negative indices—which is uniquely possible—we get

$$
M_{-k}(s)=\left(M_{k}\left(q^{-k} s\right)\right)^{-1}
$$

and therefore

$$
M_{-n}(s)=\frac{1}{d_{n}\left(q^{-n} s\right)}\left(\begin{array}{cc}
F_{n+1}^{*}\left(q^{-n} s, q\right) & -F_{n}^{*}\left(q^{-n} s, q\right) \\
-t\left(q^{-n-1} s\right) F_{n}^{*}\left(q^{-n+1} s, q\right) & t\left(q^{-n-1} s\right) F_{n-1}^{*}\left(q^{-n+1} s, q\right)
\end{array}\right)
$$

with $d_{n}(s)=(-1)^{n} t\left(\frac{s}{q}\right) t(s) t(q s) \cdots t\left(q^{n-2} s\right)$. This implies

$$
F_{-n}^{*}(s, q)=(-1)^{n-1} \frac{F_{n}^{*}\left(q^{-n} s, q\right)}{t\left(\frac{s}{q^{2}}\right) \cdots t\left(\frac{s}{q^{n+1}}\right)} .
$$

The Cassini identity is a special case of the Cassini-Euler identity

$$
F_{n-1}^{*}(q s, q) F_{n+k}^{*}(s, q)-F_{n}^{*}(s, q) F_{n+k-1}^{*}(q s, q)=(-1)^{n} t(s) \cdots t\left(q^{n-2} s\right) F_{k}^{*}\left(q^{n} s, q\right) .
$$

For the Al-Salam and Ismail polynomials this identity has been proved in Theorem 3.1 of [10]. Other special cases are mentioned in [7].

The Cassini-Euler formula is an immediate consequence of (5.3) if we write it in the form

$$
M_{k+n}(s) M_{n}(s)^{-1}=M_{k}\left(q^{n} s\right)
$$

and compare the upper right entries of the matrices.

A combinatorial proof. There is also a more illuminating combinatorial proof of this formula, inspired by [12]. Let $m \in \mathbb{Z}$ and $w=c_{1} c_{2} \cdots c_{i}$ a Morse code sequence of dots and dashes of total length $n$. If we put this sequence upon the interval $\{m, m+1, \ldots, m+n-1\}$ we say that it starts at $m$ and ends at $m+n-1$. We associate with this sequence a weight $\lambda(w)=\lambda\left(c_{1}\right) \cdots \lambda\left(c_{i}\right)$ by $\lambda(c)=x\left(q^{j} s\right)$ if $c$ is a dot at $j, \lambda(c)=t\left(q^{j} s\right)$ if $c$ is a dash starting at $j$, and $\lambda(c)=1$ if $c$ is ending at $j$. It is clear that for a Morse code sequence $w$ starting at $m$ we have $\lambda(w)=\eta^{m} \Phi_{2}(w)$.

Now consider all ordered pairs of Morse code sequences $(u, v)$ where $u$ starts at 0 and has length $n+k-1$ for some $k \geq 1$ and $v$ starts at 1 and has length $n-2$. Their total weight is $F_{n-1}^{*}(q s, q) F_{n+k}^{*}(s, q)$. If there is an $i, 0 \leq i \leq n-2$, where in one of the sequences occurs a dot, there is also a minimal $i_{\text {min }}$ with this property. Then we exchange the sequences starting at $i_{\min }+1$. Note that at this point in each of the sequences we have either a dot or the initial point of a dash, so that we really can exchange the rest of the sequences. Thus to each pair $(u, v)$ there is associated a pair $(\hat{u}, \hat{v})$ where $\hat{u}$ starts at 0 and has length $n-1$ and $\hat{v}$ starts at 1 and has length $n+k-2$.

It is clear that $\lambda(u) \lambda(v)=\lambda(\hat{u}) \lambda(\hat{v})$. The total weight of all pairs $(\hat{u}, \hat{v})$ is $F_{n}^{*}(s, q) F_{n+k-1}^{*}(q s, q)$.

The only pairs where this bijection fails are for $n$ even those where in $v$ there are only dashes and in $u$ all places up to $n-1$ are occupied by dashes. The total weight of these pairs is $t(s) t(q s) \cdots t\left(q^{n-2} s\right) F_{k}^{*}\left(q^{n} s, q\right)$.

If $n$ is odd then this bijection fails at those pairs $(\hat{u}, \hat{v})$ where $\hat{u}$ has only dashes and in $\hat{v}$ all places up to $n-1$ are occupied by dashes. Thus the $q$-Cassini-Euler formula is proved. 
For $j>2$ we consider matrices

$$
A(j, s)=\left(\begin{array}{ccccc}
0 & 1 & 0 & \ldots & 0 \\
0 & 0 & 1 & \ldots & 0 \\
\ldots & \ldots & \ldots & \ldots & \ldots \\
0 & 0 & 0 & \ldots & 1 \\
t\left(\frac{s}{q}\right) & 0 & 0 & \ldots & x(s)
\end{array}\right)
$$

and define

$$
M_{n}(j, s)=A\left(j, q^{n-1} s\right) A\left(j, q^{n-2} s\right) \cdots A(j, s)
$$

and $M_{0}(j, s)=I$, the identity matrix.

Then the $i$-th row, $0 \leq i \leq j-1$ of $M_{n}(j, s)$ is given by

$$
\left(t\left(\frac{s}{q}\right) F_{n-1+i}^{*}(q s), t(s) F_{n-2+i}^{*}\left(q^{2} s\right), \ldots, t\left(q^{j-3} s\right) F_{n-j+1+i}^{*}\left(q^{j-1} s\right), F_{n+i}(s)\right),
$$

where we only indicate the dependence on $s$.

To prove this observe that $g_{n}(k, s):=t\left(q^{k-1} s\right) F_{n-k-1}\left(q^{k+1} s\right)$ satisfies the recurrence relation of the Fibonacci polynomials with the initial conditions $g_{i}(k, s)=\delta_{i, k}$.

\section{References}

[1] W. A. Al-Salam M. E. H. Ismail, Orthogonal polynomials associated with the Rogers-Ramanujan continued fraction, Pacific. J. Math. 104 (1983), 269-283.

[2] G. E. Andrews, R. Askey, R. Roy, Special Functions, Cambridge University Press 1999.

[3] G. E. Andrews, Fibonacci numbers and Rogers-Ramanujan identities, 2002, to appear in Fibonacci Quart.

[4] L. Carlitz, Fibonacci notes 4, Fibonacci Quart. 13 (1975), 97-102.

[5] J. Cigler, Operatormethoden für q-Identitäten, Monatsh. Math. 88 (1979), 87-105.

[6] J. Cigler, Elementare q-Identitäten, Sém. Lothar. Combin. B05a (1981), http: //www. mat. univie.ac.at/ slc.

[7] J. Cigler, q-Fibonacci polynomials, Fibonacci Quart. 41 (2003), 31-40.

[8] J. Cigler, Einige q-Analoga der Lucas- und Fibonacci-Polynome, to appear in Österr. Akad. Wiss.

[9] R. F. Graham, D. E. Knuth, O. Patashnik, Concrete Mathematics, Addison-Wesley (1988).

[10] M. E. H. Ismail, H. Prodinger, D. Stanton, Schur's determinants and partition theorems, Sém. Lothar. Combin. B44a (2000), http: / / www. mat.univie.ac.at/ slc.

[11] P. Paule, H. Prodinger, Fountains, histograms, and q-identities, (2002).

[12] M. Werman, D. Zeilberger, A bijective proof of Cassini's Fibonacci identity, Discrete Math. 58 (1986), 109. 
Johann Cigler 